\title{
Sleep architecture and cognitive changes in olanzapine-treated patients with depression: A double blind randomized placebo controlled trial
}

\author{
Lauren K Lazowski ${ }^{1}$, Ben Townsend ${ }^{2}$, Emily R Hawken ${ }^{1,3}$, Ruzica Jokic ${ }^{3}$, Regina du Toit ${ }^{3}$ and Roumen Milev ${ }^{3 *}$
}

\begin{abstract}
Background: Disturbance in sleep quality is a symptom of Major Depressive Disorder (MDD) and Bipolar Disorder (BD) and thus improving quality of sleep is an important aspect of successful treatment. Here, a prospective, double-blind, randomized, placebo-controlled study examined the effect of olanzapine (an atypical antipsychotic) augmentation therapy on sleep architecture, specifically slow wave sleep (SWS), in the treatment of depression. The effect of olanzapine augmentation therapy on other features of sleep (e.g., sleep continuity) and depression (e.g., illness severity and cognitive function) were also determined.
\end{abstract}

Methods: Patients currently experiencing a major depressive episode and who were on a stable medication were included. Sleep architecture was measured by overnight ambulatory polysomnography. Illness severity was determined using the Montgomery-Asberg Depression Rating Scale (MADRS). Cognitive function was examined using Cambridge Neuropsychological Test Automated Battery (CANTAB): Spatial Working Memory (SWM), Spatial Span (SSP), and Reaction Time (RTI) tasks. Polysomnographs, clinical measures and cognitive tests were administered at baseline, after 2-4 days of treatment and after 28-31 days of treatment. Twenty-five patients participated in the study ( $N=10, N=15$ for placebo and olanzapine treated groups respectively).

Results: The primary objective of the study was to assess the objective (polysomnographic) changes in sleep quality, defined as changes in SWS, following olanzapine treatment for depression. Latency to but not duration of SWS was found to significantly differ between olanzapine- and placebo-treated participants (Hedge's g: 0.97, 0.13 respectively). A significant improvement in olanzapine-treated participants over placebo-treated participants was observed in secondary outcome measures, including sleep efficiency, total sleep time, and sleep latency. Secondary objectives assessed the subjective changes in sleep quality parameters and correlated them with measures of illness severity and changes in cognition. MADRS scores were significantly improved in olanzapine-treated participants over time but not more than placebo treatment. There was no significant difference between olanzapine- and placebo-treated participants in SWM, SSP or RTI tasks.

Conclusions: Olanzapine augmentation treatment generally did not improve SWS but did improve sleep continuity and depression. Olanzapine may be one of few medications that improve sleep continuity, thus directly targeting symptoms of depression.

Trial registration: ClinicalTrials.gov, NCT00520507.

Keywords: Olanzapine, Sleep continuity, Slow-Wave sleep, Depression, Cognitive function, Illness severity

\footnotetext{
* Correspondence: roumen.milev@queensu.ca

${ }^{3}$ Department of Psychiatry, Queen's University, 752 King Street West,

Kingston, ON K7L 4X3, Canada

Full list of author information is available at the end of the article
} 


\section{Background}

Disturbance in sleep quality is a symptom of Major Depressive Disorder (MDD) and Bipolar Disorder (BD) [1], with MDD having the strongest association with sleep disturbances [2,3]. It is well documented that the severity of the sleep disturbance is related to treatment response, as well as the chronicity of the disorders [4,5], and as such, is an important aspect of treatment.

Sleep has two main components: Rapid Eye Movement (R) and Non-REM (N) sleep [6]. N sleep includes stages 1 to 3 , where stage 3 is referred to as slow wave sleep (SWS). SWS is considered the deepest stage of sleep and involves a higher arousal threshold [7]. This is the most prevalent stage of the sleep cycle in the first third of the night, approximately $10 \%-15 \%$ of total sleep time (TST). As SWS shortens in each cycle, the amount of R sleep increases, with $\mathrm{R}$ sleep being most prevalent during each cycle in the final third of the night, constituting 20\%-25\% of TST [6].

Sleep architecture in patients experiencing depression is generally thought to be altered [8]. Sleep during depressive episodes is characterized by a reduction in SWS, or increased latency to SWS, increased duration of $R$ sleep, shortened latency to $R$ sleep, continuity disturbances, and decreased TST [9-12]. Altered SWS and R sleep are seen in approximately $50 \%$ of patients with affective disorders [12]. Sharpley et al. [13], found a negative association between SWS activity and illness severity of depression; however, variables such as sleep efficiency and TST were also found to significantly increase as illness severity decreased.

Cognitive deficits in MDD and BD include working memory, attention and psychomotor speed [14-18]. Acute and chronic sleep deprivation has been shown to affect attention, working memory and cognitive function [19-22]. In healthy volunteers, SWS deprivation was associated with impaired cognitive performance and a delayed reaction time upon awakening [23,24]. In particular, studies have shown that duration of SWS per night is correlated with working memory performance in elderly and schizophrenic samples [25,26]. However, the role of sleep in the cognitive performance of patients with mood disorders is unknown.

Monoaminergic neurotransmitters are thought to be involved in the relationship between sleep and depression. The serotonergic [27,28], cholinergic [6], histaminergic [29,30], and noradrenergic [27] systems all contribute to the pathophysiology of depression and the underlying mechanisms of sleep. Antidepressants can produce changes in the sleep electroencephalogram (EEG) of depressed patients, the most prominent being the suppression of $\mathrm{R}$ sleep. This is thought to occur by increasing norepinephrine (NA) and/or serotonin (5-HT) function [31]. 5-HT receptors have also been shown to play a critical role in the regulation of SWS. Several antidepressants, particularly tricyclic antidepressants and trazodone, are thought to normalize SWS because of their affinity for $5-\mathrm{HT}_{2 \mathrm{~A}}$ and $5-\mathrm{HT}_{2 \mathrm{C}}$ receptors [31].

It has been estimated that up to $30 \%$ of patients with depression fail to respond to traditional antidepressant treatments alone [32]. Augmentation of antidepressant therapy, with olanzapine, an atypical antipsychotic, is often used in routine clinical practice for the treatment of depression. Olanzapine has affinity for dopamine $\mathrm{D}_{1}, \mathrm{D}_{3}$, and $\mathrm{D}_{4}$; serotonin 5- $\mathrm{HT}_{2 \mathrm{~A} / \mathrm{C}}, 5-\mathrm{HT}_{3}$ and 5-HT-6; muscarinic $\mathrm{M}_{1}-\mathrm{M}_{5}$; adrenergic $\alpha_{1}$; and histamine $\mathrm{H}_{1}$ receptors. Moreover, olanzapine is an antagonist at these sites, with highest affinity for both the dopamine and serotonin receptors [33].

To date, many studies have focused on improving illness severity of patients with MDD or BD by combining mood stabilizing medication with a change in sleeprelated behaviours [34-36]. A review by Riemann et al. [8] described several different interventions used to improve sleep habits of patients with MDD, such as psychotherapy [37], pharmacological treatment [38], and sleep deprivation therapy [39], among others. A large majority of these studies, regardless of treatment type, found that improving sleep continuity measures resulted in improvements in mood. Olanzapine has been shown to increase sleep continuity, subjective sleep measures and SWS in healthy volunteers [40-42] while an association between changes in sleep architecture and mood improvement in patients with MDD remains unclear [13]. Finally, Olanzapine has also been shown to improve cognition in schizophrenic patients [43]; however, its impact on cognition in depressive patients has not yet been fully described.

The primary aim of this study was to examine the effects of olanzapine augmentation treatment on sleep architecture, specifically SWS, in patients experiencing a major depressive episode. Furthermore, we examined several secondary outcome measures to determine the effects of olanzapine augmentation on other elements of sleep, like TST and sleep continuity, and aspects of depression, including illness severity and cognitive function.

\section{Methods}

The present study was a prospective, double-blind, placebo-controlled, repeated measures, polysomnographic study. This study was approved by the Queen's University research ethics board, Health Canada, and was registered with clinicaltrials.gov (NCT00520507).

\section{Participants}

A $15 \%$ change in the primary outcome measure, SWS, was selected to be a clinically significant and meaningful 
change; this effect size was used to calculate sample size, indicating that 30 participants ( 15 placebo, 15 treatment) were needed to have enough power to detect an effect of olanzepine on SWS. To account for drop-outs, we aimed to enroll 40 participants. The trail was stopped, however, before reaching recruitment goals (May 2009) due to an inability to recruit participants. Only thirty-one participants signed informed consent to participate in this study.

Participants were screened and enrolled into the study (beginning October 2007) by blinded clinicians (nurses) and recruited from tertiary care mood disorders units, general practitioners offices, and from the community. All were 18 years of age or older, and met DSM-IV-TR criteria [44] for MDD, Bipolar I Disorder, Bipolar II Disorder or Bipolar Disorder NOS, as confirmed by the Mini International Neuropsychiatric Inventory (MINI) [45]. At enrollment, participants were experiencing a major depressive episode (MDE), defined as a Hamilton Depression Rating Scale-17 item (HDRS-17) score of $>15$, and not a mixed episode, defined as a Young Mania Rating Scale Score (YMRS) of $\leq 12$ [46]. Excluded criteria were current or past diagnosis of schizophrenia or dementia, substance abuse within three months of enrolment (excluding caffeine or nicotine), imminent risk of suicide or danger to themselves or others, known intolerance for olanzapine, a serious or inadequately treated medical illness, a history of seizures, previous enrollment in the study or enrollment in another treatment study within 4 weeks prior. Participants could not be taking any other antipsychotic medication at the time of enrolment and must have been on a stable dose of all medications for 4 weeks prior to enrolment. Benzodiazepines and other sleep aids were discontinued if a stable dose had not been achieved for four weeks prior to enrolment (number of participants on each concomitant drug: Placebo: 1 Remeron, 2 Prozac, 1 Valproic Acid, 2 Clonazepam, 1 Lorazepam, 1 Trazodone, 1 Citalopram, 1 bupropion, 1 fluvoxamine, 1, cipralex, 1 oxycontin, 1 Ativan, 1 Topamax, 1 fluvianzine; Olanzepine: 2 Remeron, 2 Prozac, 1 doxepine, 1 amitriptyline, 1 oxazepam, 5 Clonazepam, 1 ritalin, 1 Lorazepam, 1 lithium, 1 immovane 1 Citalopram, 2 bupropion, 1 fluvoxamine, 2 cipralex, 2 Effexor). Four participants failed baseline screening ( 1 had a HAMD score that was too low, 1 was already receiving olanzepine treatment [and also had a too-low HAMD score], and 2 failed to show at the screening appointment) and two participants withdrew (refused to continue to participate) between randomization and day 2-4, and were not included in the analysis. Thus, 25 participants were included in the analysis. Three participants from the olanzapine-treated group terminated from the study before day 28-31, due to a worsening of original mood symptoms. Participants were monitored for sleep apnea and/or hypopnea throughout the course of the study as the presence of these conditions at any point warranted exclusion from the study due to the confounding nature of these events on sleep architecture. No participants needed to be removed due to obstructive sleep apnea/hypopnea.

\section{Clinical measures}

Participants were assessed at three time-points by a clinician blinded to treatment group: baseline (before randomization and administration of study medication), 2-4 days, and 28-31 days after the administration of study medication. Each clinical assessment consisted of the HDRS-17 [47], the Montgomery Asberg Depression Rating Scale (MADRS) [48], the Hamilton Anxiety Rating Scale (HARS) [49,50], the YMRS [46], the Pittsburgh Sleep Quality Index (PSQI) [51], Visual Analogue Scale (VAS) for sleep quality [52], and the Epworth Sleepiness Scale (ESS) [53]. At baseline, the MINI and the Clinical Global Impression-Severity (CGI-S) [54], scales were administered. During day 28-31, the Clinical Global Impression-Improvement (CGI-I) was administered. Baseline blood work, physical exam, and pregnancy test (in women of childbearing potential) were performed.

\section{Neurocognitive measures}

At each visit, participants completed cognitive testing using three Cambridge Neuropsychological Test Automated Battery (CANTAB) tasks, including: Spatial Span (SSP), Spatial Working Memory (SWM) and Reaction Time (RTI) [55,56] (Cambridge Cognition, Cambridge, UK; www.camcog.com). One participant did not complete the CANTAB testing.

\section{Medication}

Participants were randomly assigned to either placebo or olanzapine (orally disintegrating, zydis, formulation) conditions. A randomization table was generated by a statistician to randomize for order that then placed sealed envelopes containing a single allocation (A:Placebo, B:Olanzapine) into a box in the order specified by the randomization table. Following screening, the participant was instructed to pull the next envelope in the box. This allocation was given to an unblinded pharmacist who filled a prescription that read 'placebo/olanzapine.' Medication dosing started at $2.5 \mathrm{mg}$ on day 1 and increased to $5 \mathrm{mg}$ at day 2 ; during day $2-4$, the dosing was titrated up or down, in increments of $2.5 \mathrm{mg}$ to a maximum of $20 \mathrm{mg}$. The mean dose of olanzapine was $6.67 \mathrm{mg}$ at the end of the study; doses ranged from $5 \mathrm{mg}$ to $10 \mathrm{mg}$. Eight participants in the placebo-treated group and twelve in the olanzapinetreated group were taking at least one antidepressant as concomitant medications; concomitant antidepressants 
included amitriptyline, bupropion, escitalopram, citalopram, doxepine, venlafaxine, fluoxetine, remeron, and trazodone. One participant in the placebo-treated group was taking only a benzodiazepine; one participant in the olalnzapine-treated group was only taking one mood stabilizer; one participant in the placebotreated group and two in the olanzapine-treated group were not taking any other psychotropic medications. All concomitant medications were stable throughout the duration of the study.

\section{Polysomnographic recordings}

At each time-point, an overnight sleep polysomnograph (PSG) was performed by a clinician blinded to treatment group at the participants' home, using the MediPalm Personal Recording Device (Braebon Medical Corporation, Kanata, Ontario, Canada). The overnight sleep PSG included four electroencephalogram channels (C4-A1, C3-A2, O2-A1, O1-A2 [57]), an electro-oculogram (two channels), a submental electromyogram (EMG), a finger pulse oximetry, an oronasal airflow (oronasalthermistor), a chest and abdominal movement belt (respiratory inductance plethysmography), a vibration snore sensor and an anterior tibialis EMG. A position sensor was used to monitor position continuously (Ultima Body Position Sensor; Braebon Medical Corporation, Carp, Canada). Participants were not monitored in person. Sharpley et al. $[31,58,59]$ have demonstrated that the use of home sleep recordings provides a reliable means of detecting the effects of medications on sleep architecture. Once the PSG was applied (approximately $1900 \mathrm{hrs}$ each study night), a timer was set to record for eight hours following usual sleep time or until the participant rose in the morning. Usual sleep time was verbally confirmed for each participant. Participants were asked to retire and rise at their usual time and were to refrain from alcohol on study nights; however, normal caffeine and nicotine intake was maintained. While participants did not all go to bed at the same time, eight hours or less was sufficient sleep time for those enrolled. Clinicians returned in the morning (following the participants' usual wake time) to retrieve the PSG equipment. Sleep latencies were calculated from lights out. PSGs were manually scored in 30-second epochs according to standardized criteria of American Academy of Sleep Medicine [60], using Pursuit Advanced Sleep System software (Braebon Medical Corporation, Carp, Canada). Ruehland et al. [61] have demonstrated that measures of sleep architecture are not affected by the number of EEG electrode placements used in scoring the PSG. Technicians were blinded to treatment status. Only one PSG recordings was excluded from analysis due to technical problems. Obstructive apneas and hypopneas, defined as partial airway obstructions leading to a 50\% reduction in thoracoabdominal movement lasting for at least 10 seconds [62], were scored using the criteria from the American Academy of Sleep Medicine Task Force [63]. Arousals were scored based on American Sleep Disorders Association criteria, 1992 [57]. The respiratory disturbance index (RDI), which included apneas, hypopneas, and snore arousals for the number of events per hour of sleep, were calculated.

\section{Statistical analysis}

PSG recording and clinical measures (with the exception of the CGI) were analyzed using 2-way and 1-way repeated measures analysis of variance (ANOVA). The design included two treatment groups (placebo $\times$ olanzapine) across three time-points (baseline, Day 2-4 and Day 28-31). Independent-samples t-tests examined post-hoc comparisons. Hedge's g was used to calculate the effect size of the primary outcome measure, changes in SWS, between groups on Day 28-31. Missing PSG, clinical and CANTAB data were replaced with the last observation carried forward. One participant did not complete any of the CANTAB and was not included for this analysis. One-tailed distributions were used for all clinical measures, while for polysomnographic and neurocognitive measures, two-tailed distributions were used. Additionally, demographic comparisons between groups were analyzed using Fisher's exact tests or ANOVAs (see Table 1). For repeated-measures ANOVA, trend analyses (contrasts) were performed and reported. Neither sphericity of variance nor a significant main effect of the within-subject variable is an assumption of running trend analysis.

\section{Results}

Twenty-five participants were included in the analysis ( $\mathrm{N}=15$ olanzepine and $\mathrm{N}=10$ placebo treated). The mean $( \pm \mathrm{SD})$ age of the olanzapine-treated group and placebo-treated group was $46 \pm 17$ years (range: $20-59$,

Table 1 Sociodemographic characteristics

\begin{tabular}{|c|c|c|c|c|}
\hline & & $\begin{array}{l}\text { Placebo group } \\
\quad(\mathrm{N}=10)\end{array}$ & $\begin{array}{l}\text { Olanzapine group } \\
\qquad(N=15)\end{array}$ & \\
\hline & & $\mathrm{N}$ & $\mathrm{N}$ & P-value \\
\hline \multirow[t]{3}{*}{ Gender (\%) } & & & & 0.69 \\
\hline & Male & $5(50)$ & $6(40)$ & \\
\hline & Female & $5(50)$ & $9(60)$ & \\
\hline \multirow[t]{3}{*}{ Diagnosis (\%) } & & & & 1.0 \\
\hline & MDD & $5(50)$ & $7(47)$ & \\
\hline & $\mathrm{BD}$ & $5(50)$ & $8(53)$ & \\
\hline Age $( \pm S D)$ & & $46(9)$ & $46(17)$ & 0.24 \\
\hline \multirow[t]{2}{*}{ Weight $( \pm S D)$} & Visit 1 & $185(50)$ & $178(36)$ & 0.76 \\
\hline & Visit 4 & $185(51)$ & $182(37)$ & \\
\hline
\end{tabular}

MDD = Major Depressive Disorder, BD = Bipolar Disorder. P-value reported for the test statistic for Fisher's Exact Test (gender and diagnosis) and between groups ANOVA and repeated measures ANOVA (age and weight, respectively). 
median: 45) and $46 \pm 9$ years (range: $19-79$, median: 46), respectively and did not differ significantly between the treatment groups (see Table 1).

\section{Polysomnographic measures}

Table 2 summarizes all primary and secondary polysomnographic outcome measures for sleep architecture, in both olanzapine and placebo groups, across baseline, day 2-4, and day 28-31. Figure 1 shows the percentage of TST spent in SWS and latency to SWS in both olanzapine- and placebo-treated groups. A 2-way repeated measures ANOVA did not yield significant changes in percentage of TST spent in SWS between the olanzapine- or placebotreated group $(\mathrm{F}[1,23]=2.36 ; \mathrm{p}=0.138$; Hedge's $\mathrm{g}=0.53)$ across time $(\mathrm{F}[1,23]=0.256 ; \mathrm{p}=0.775)$. Latency to SWS significantly decreased with olanzapine treatment by the end of the trial (no main effect of treatment, $F[1,23]=2.49$, $\mathrm{p}=.128$; significant interaction of time $\mathrm{x}$ treatment, $\mathrm{F}[1,23]=5.49, \mathrm{p}=0.028)$. Comparing the two treatment groups at each time point indicated that at the beginning of the trial latency to SWS was not different $(\mathrm{t}[23]=-.192$, $\mathrm{p}=.849)$ but by Day $28-31$, the olanzapine treated group reached SWS faster than the placebo group $(\mathrm{t}[23]=2.42$, $p=0.024$; hedges $g=0.97$ ). Latency to sleep however, was not significantly different and could not account for decreased latency to SWS in the olanzapine treated group (no main effect of treatment $F[1,23]=2.17, \mathrm{p}=0.154$ or time $F[1,23]=0.297, p=.591$ ). Duration of SWS was also not significantly affected by the olanzapine treatment compared to placebo $(\mathrm{F}[1,23]=0.581 ; \mathrm{p}=0.454)$ over time $(\mathrm{F}[1,23]=0.00 ; \mathrm{p}=0.984$; Hedge's $\mathrm{g}=0.13)$.

TST and Sleep efficiency are shown in Figure 2 for both olanzapine- and placebo-treated groups. Two twoway repeated-measures ANOVA showed an increase in TST (trend for main effect of treatment, $F[1,23]=3.69$, $\mathrm{p}=0.067$ ) and sleep efficiency (main effect of treatment, $\mathrm{F}[1,23]=5.10, \mathrm{p}=0.034)$ of the olanzapine-treated group compared to the placebo-treated group. Both TST and sleep efficiency demonstrated significant time $\mathrm{x}$ treatment interactions (TST: $\mathrm{F}[1,23]=4.58 ; \mathrm{p}=.043$; sleep efficiency: $\mathrm{F}[1,23]=5.27, \mathrm{p}=0.031)$. Post-hoc independent-samples t-tests were conducted to analyze the nature of these

Table 2 Polysomnographic measures

\begin{tabular}{|c|c|c|c|c|c|c|c|}
\hline \multirow[t]{3}{*}{ Sleep parameter } & \multicolumn{3}{|c|}{ Placebo } & \multicolumn{4}{|c|}{ Olanzapine } \\
\hline & Baseline & Day 2-4 & Day 28-31 & Baseline & Day 2-4 & Day 28-31 & ANOVA \\
\hline & \multicolumn{3}{|c|}{ Mean \pm SD } & \multicolumn{4}{|c|}{ Mean \pm SD } \\
\hline \multicolumn{8}{|l|}{ Sleep continuity } \\
\hline Total time in bed (min) & $468 \pm 27$ & $454 \pm 37$ & $458 \pm 30$ & $460 \pm 41$ & $457 \pm 32$ & $461 \pm 32$ & $p=.523$ \\
\hline Total sleep time (min) & $315 \pm 62$ & $307 \pm 58$ & $289 \pm 75$ & $326 \pm 168$ & $383 \pm 66$ & $363 \pm 89$ & $p=.043$ \\
\hline Total wake time (min) & $153 \pm 67$ & $148 \pm 63$ & $169 \pm 75$ & $135 \pm 107$ & $74 \pm 68$ & $80 \pm 63$ & $p=.023$ \\
\hline No. of awakenings & $29 \pm 18$ & $23 \pm 9$ & $26 \pm 11$ & $18 \pm 7.0$ & $13 \pm 4.9$ & $15 \pm 7.1$ & $p=.775$ \\
\hline No. of stage changes & $145 \pm 77$ & $125 \pm 50$ & $123 \pm 50$ & $99 \pm 36$ & $101 \pm 44$ & $98 \pm 38$ & $p=.254$ \\
\hline Respiratory disturbance index & $15 \pm 14$ & $13 \pm 12$ & $8.7 \pm 7.2$ & $13 \pm 21$ & $11 \pm 25$ & $12 \pm 25$ & $p=.126$ \\
\hline Sleep efficiency & $67 \pm 14$ & $68 \pm 14$ & $63 \pm 17$ & $71 \pm 23$ & $84 \pm 14$ & $83 \pm 13$ & $p=.031$ \\
\hline \multicolumn{8}{|l|}{ Sleep architecture } \\
\hline Latency to sleep (min) & $76 \pm 61$ & $86 \pm 67$ & $92 \pm 78$ & $69 \pm 83$ & $38 \pm 50$ & $41 \pm 45$ & $p=0.069$ \\
\hline Latency to Stage 1 (min) & $67 \pm 54$ & $73 \pm 51$ & $85 \pm 77$ & $72 \pm 74$ & $42 \pm 42$ & $45 \pm 41$ & $p=.073$ \\
\hline Duration of Stage 1 (min) & $36 \pm 28$ & $30 \pm 16$ & $31 \pm 16$ & $32 \pm 18$ & $25 \pm 12$ & $27 \pm 10$ & $p=.984$ \\
\hline Duration of Stage 1 (\% of TST) & $11 \pm 8.0$ & $10 \pm 4.5$ & $11 \pm 5.0$ & $11 \pm 11$ & $6.5 \pm 2.8$ & $7.2 \pm 3.5$ & $p=.353$ \\
\hline Latency to Stage 2 (min) & $82 \pm 68$ & $92 \pm 72$ & $96 \pm 81$ & $79 \pm 86$ & $46 \pm 59$ & $47 \pm 49$ & $p=.058$ \\
\hline Duration of Stage 2 (min) & $168 \pm 62$ & $160 \pm 62$ & $166 \pm 58$ & $199.3 \pm 69.3$ & $230.7 \pm 55.4$ & $230 \pm 55$ & $p=.048$ \\
\hline Duration of Stage 2 ( $\%$ of TST) & $53 \pm 13$ & $51 \pm 12$ & $56 \pm 12$ & $57 \pm 12$ & $60 \pm 12$ & $60 \pm 10$ & $p=.927$ \\
\hline Latency to R (min) & $212 \pm 88$ & $220 \pm 89$ & $277 \pm 250$ & $165 \pm 132$ & $184 \pm 100$ & $180 \pm 97$ & $p=.552$ \\
\hline Duration of R (min) & $59 \pm 20$ & $66 \pm 27$ & $48 \pm 28$ & $77 \pm 50$ & $82 \pm 37$ & $87 \pm 39$ & $p=.285$ \\
\hline Duration of R (\% of TST) & $19 \pm 5.9$ & $22 \pm 7.8$ & $16 \pm 7.5$ & $20 \pm 12$ & $21 \pm 7.7$ & $22 \pm 8.4$ & $p=.239$ \\
\hline Latency to SWS (min) & $112 \pm 75$ & $116 \pm 14$ & $172 \pm 124$ & $121 \pm 122$ & $59 \pm 64$ & $77 \pm 70$ & $p=.028$ \\
\hline Duration of SWS (min) & $48 \pm 34$ & $47 \pm 31$ & $43 \pm 27$ & $28 \pm 25$ & $44 \pm 48$ & $38 \pm 43$ & $p=.295$ \\
\hline Duration of SWS (\% of TST) & $17 \pm 13$ & $18 \pm 13$ & $17 \pm 14$ & $10 \pm 12$ & $12 \pm 12$ & $10 \pm 12$ & $p=.994$ \\
\hline
\end{tabular}

The mean \pm standard deviation (SD) of sleep parameters for both olanzapine and placebo treated group. TST = Total Sleep Time, SWS = Slow Wave Sleep, REM = Rapid Eye Movement. $P$ value reported for the interaction (time by treatment) of a two-way repeated measures ANOVA. 

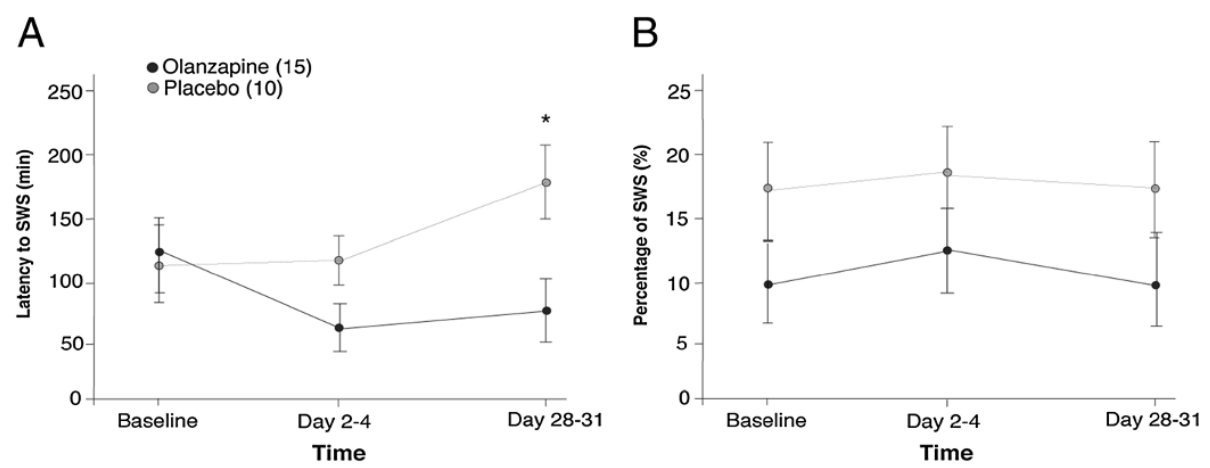

Figure 1 Latency to slow wave sleep and percentage of slow wave sleep. A) Mean \pm standard error of the mean for latency to slow wave sleep (SWS) in minutes, for both olanzapine- and placebo-treated groups for each time. B) Percentage of SWS measured by ratio of SWS within total sleep time (TST) in minutes. ${ }^{*} p=0.024$ between placebo and olanzapine groups on Day 28-31.

interactions and revealed no significant difference in TST or sleep efficiency at baseline (TST: $\mathrm{t}_{23}=-0.325, \mathrm{p}=0.798$; Sleep efficiency: $t_{23}=-0.460, p=0.65$ ) but by Day $28-31$ olanzapine treatment significantly increased both (TST: $t_{23}=-2.21 ; p=0.037$; Sleep efficiency: $t_{23}=-3.30$; $\mathrm{p}=0.003)$.

As well as an increase in TST, olanzapine treated participants experienced significantly fewer awakenings (Table 2) and less overall time awake compared to treatment with placebo (2-way repeated measures ANOVA; main effect of treatment, number of awakenings, $F[1,23]=10.0$, $\mathrm{p}=0.004$; awake time, $\mathrm{F}[1,23]=4.8, \mathrm{p}=0.037$ ). Post-hoc analysis of the significant interaction of time by treatment in awake time $(\mathrm{F}[1,23]=4.93, \mathrm{p}=0.037)$ indicated that at baseline, awake times did not differ between placebo and olanzapine-treated participants $\left(t_{23}=0.531, \mathrm{p}=0.601\right)$ but by Day 28-31, olanzapine treatment was significantly decreasing amount of awake time participants experienced $\left(\mathrm{t}_{23}=2.26, \mathrm{p}=0.003\right)$.

Two-way repeated measures ANOVA also analyzed the percentage of TST spent in $\mathrm{R}$ and duration of $\mathrm{R}$ (Table 2). There was neither a main effect of treatment or time on percentage of TST spent in $\mathrm{R}(\mathrm{F}[1,23]=.796$, $\mathrm{p}=0.382 ; \mathrm{F}[1,23]=.025, \mathrm{p}=0.875$, respectively. There was a significant effect of olanzapine treatment on duration of $\mathrm{R}(\mathrm{F}[1,23]=4.78, \mathrm{p}=0.039)$ however there was neither a significant effect of time $(F[1,23]=.003$, $\mathrm{p}=0.957)$ or a significant interaction of treatment by time $(\mathrm{F}[1,23]=.696, \mathrm{p}=0.413)$. Furthermore, Table 2 means indicate that the olanzapine group showed longer $\mathrm{R}$ than the placebo group even at baseline, suggesting that the difference in $\mathrm{R}$ sleep was not due to the addition of olanzapine.

\section{Measures}

Clinical measures are included as results of a secondary objective of the study. Figure 3 shows the mean MADRS total scores for both olanzapine- and placebo-treated participants. Two-way repeated ANOVA showed no significant differences between olanzapine and placebo treatment for MARDS or HDRS scores $(\mathrm{F}[1,23]=1.09$, $\mathrm{p}=.306 ; \mathrm{F}[1,23]=0.269, \mathrm{p}=.609$, respectively). However, both MARDS and HDRS scores significantly decreased with time $(\mathrm{F}[1,23]=6.03, \mathrm{p}=.022 ; \mathrm{F}[1,23]=20.0, \mathrm{p}=<.001$,
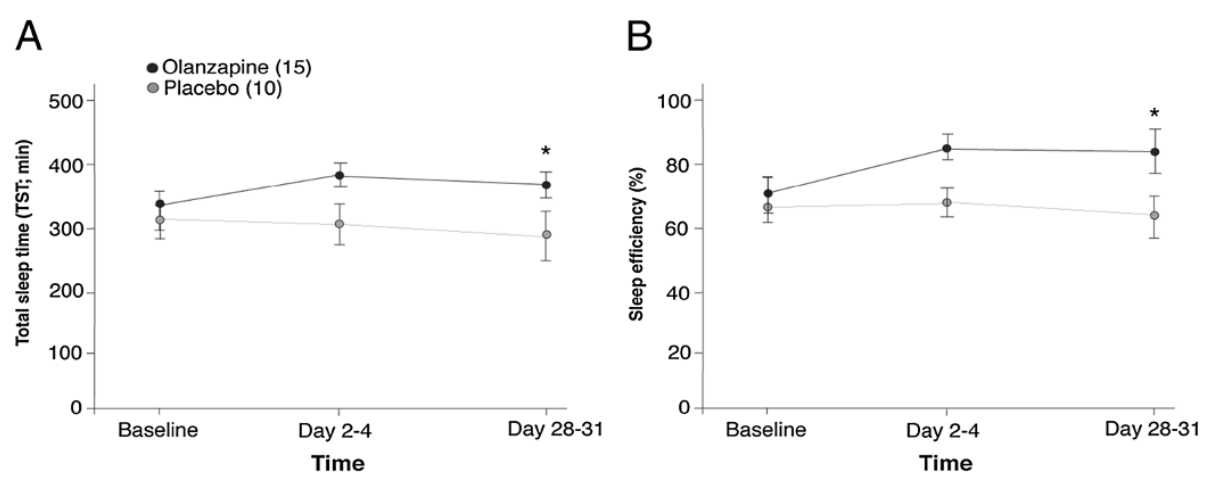

Figure 2 Sleep continuity. A) Total sleep time B) Sleep efficiency. Mean \pm standard error of the mean for both olanzapine- and placebo-treated groups. Total sleep time is measured in minutes. Sleep efficiency: $\%=$ total sleep time(TST)/time in bed (TIB) $\times 100 .{ }^{*} \mathrm{p}<.05$ between placebo and olanzapine groups on Day 28-31. 


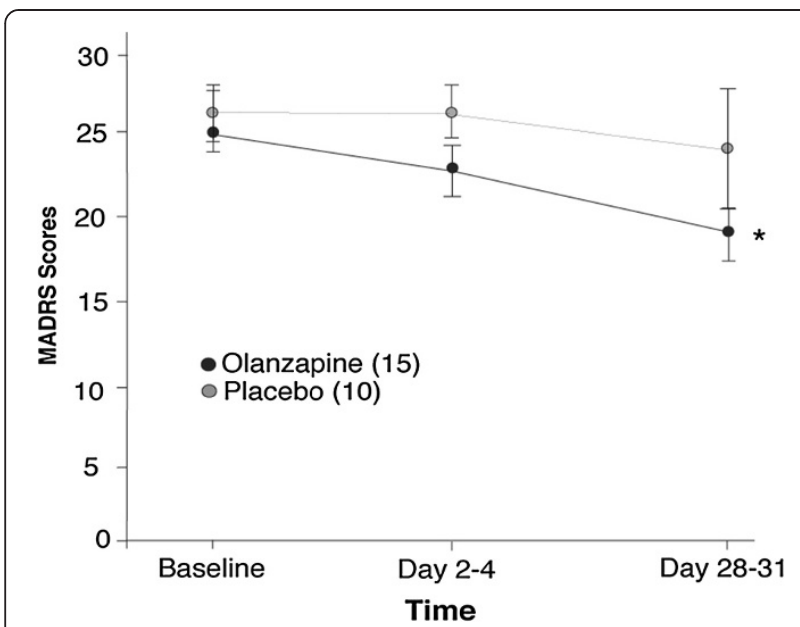

Figure 3 Montgomery-Asberg Depression Rating Scale. Total score with mean \pm standard error of the mean for both olanzapine- and placebo-treated groups. ${ }^{*} p<.001$ for olanzapine groups across time.

respectively). One-way repeated measures ANOVA showed a significant decrease in total MADRS score from baseline to the end of the trial (day 28-31) for olanzapine-treated patients (main effect of time: $\mathrm{F}$ $[1,14]=6.03, p=.022)$, where as placebo treatment did not show a decrease $(F[1,9]=0.529, \mathrm{p}=.486)$. HDRS scores also significantly decreased over time in the olanzapine-treated group $(\mathrm{F}[1,14]=19.6, \mathrm{p}=.001)$, but the placebo-treated group did not $(\mathrm{F}[1,9]=4.67, \mathrm{p}=.059)$. Table 3 shows clinician administered and self report rating scale scores for both the olanzapine- and placebo-treated groups.

\section{Neurocognitive measures}

Neurocognitive measures, evaluated as mean CANTAB testing scores, are shown in Table 4 and were assessed as a secondary objective of the study. The between errors and strategy score of the CANTAB SWM task of both the olanzapine- and placebo-treated groups did not significantly change between groups $(F[1,22]=0.3$, $\mathrm{p}=0.590 ; \mathrm{F}[1,22]=0.658, \mathrm{p}=0.426$, respectively) or across time $(\mathrm{F}[1,22]=1.4, \mathrm{p}=0.242 ; \mathrm{F}[1,22]=0.713, \mathrm{p}=0.407$, respectively). The mean spatial span length recalled on the CANTAB SSP task was also not significantly different between treatment groups or across time (2-way repeated measures ANOVA, $F[1,22]=0.102, \mathrm{p}=0.753$; $\mathrm{F}[1,22]=0.036, \mathrm{p}=0.852$, respectively). Finally, the mean reaction time and movement time from the CANTAB RTI task for both the olanzapine- and placebo-treated groups also showed no significant changes as a result of olanzapine treatment (2-way repeated measures ANOVA reaction time and movement: treamtment, $F[1,22]=0.089, \mathrm{p}=.768 ; \mathrm{F}[1,22]=0.15, \mathrm{p}=.702$, respectively) or over time (2-way repeated measures ANOVA reaction time and movement: time, $F[1,22]=2.28$, $\mathrm{p}=.145 ; \mathrm{F}[1,22]=2.57, \mathrm{p}=.123$, respectively).

\section{Discussion}

Surprisingly, the addition of olanzapine to the current medication regimes of patients experiencing either unipolar or bipolar major depressive episodes only resulted in significant improvements in latency to SWS but not in other SWS measures, the primary outcome measure of this study. However, olanzapine augmentation did significantly improve the secondary outcome measure sleep continuity, that included sleep efficiency, number of awakenings, time spent awake and total sleep time. These improvements did not extend to significant changes other aspects of sleep architecture, like percentage of $\mathrm{R}$ sleep. Treatment with olanzapine also significantly improved illness severity without changes in measures of executive and psychomotor function.

To date, Sharpley et al. [13] have reported the only study to investigate the addition of olanzapine to SSRI treatment on overnight polysomnograph in patients with

Table 3 Clinical measures

\begin{tabular}{|c|c|c|c|c|c|c|c|}
\hline \multirow{3}{*}{$\begin{array}{l}\text { Clinical } \\
\text { measures }\end{array}$} & \multicolumn{3}{|c|}{ Placebo } & \multicolumn{4}{|c|}{ Olanzapine } \\
\hline & Baseline & Day 2-4 & Day 28-31 & Baseline & Day 2-4 & Day 28-31 & ANOVA \\
\hline & \multicolumn{3}{|c|}{ Mean \pm SD } & \multicolumn{4}{|c|}{ Mean \pm SD } \\
\hline MADRS total & $26 \pm 6.3$ & $26 \pm 7.1$ & $24 \pm 3.2$ & $25 \pm 5.8$ & $23 \pm 6.2$ & $19 \pm 11$ & $p=.175$ \\
\hline HDRS total & $21 \pm 5.5$ & $19 \pm 5.9$ & $18 \pm 7.4$ & $22 \pm 5.0$ & $18 \pm 6.4$ & $15 \pm 8.4$ & $p=.123$ \\
\hline HARS total & $16 \pm 2.9$ & $13 \pm 4.7$ & $13 \pm 5.3$ & $17 \pm 5.8$ & $15 \pm 7.4$ & $13 \pm 6.7$ & $p=.420$ \\
\hline YMRS total & $3.9 \pm 3.4$ & $3.7 \pm 2.5$ & $4.7 \pm 4.2$ & $3.7 \pm 3.1$ & $2.9 \pm 3.0$ & $3.5 \pm 2.3$ & $p=.223$ \\
\hline PSQI total & $13 \pm 3.0$ & $14 \pm 3.6$ & $12 \pm 5.2$ & $12 \pm 3.5$ & $11 \pm 4.2$ & $9.9 \pm 4.7$ & $p=.699$ \\
\hline VAS total & $31 \pm 15$ & $32 \pm 19$ & $43 \pm 27$ & $37 \pm 27$ & $45 \pm 28$ & $59 \pm 30$ & $p=.415$ \\
\hline ESS total & $7.4 \pm 6.2$ & $5.4 \pm 4.2$ & $5.8 \pm 5.9$ & $8.4 \pm 5.2$ & $8.5 \pm 5.6$ & $7.3 \pm 5.2$ & $p=.666$ \\
\hline
\end{tabular}

The mean \pm standard deviation (SD) of selected clinical measures for both olanzapine and placebo treated group. MADRS = Montgomery Asberg Depression Rating Scale, HDRS = Hamilton Depression Rating Scale, HARS = Hamilton Anxiety Rating Scale, YMRS = Young Mania Rating Scale, VAS = Visual Analogue Scale for sleep quality, PSQI = Pittsburg Sleep Quality Index, ESS = Epworth Sleepiness Scale. P value reported for the interaction (time by treatment) of a two-way repeated measures ANOVA. 
Table 4 Cognitive measures

\begin{tabular}{|c|c|c|c|c|c|c|c|}
\hline \multirow{3}{*}{$\begin{array}{l}\text { Cognitive } \\
\text { measures }\end{array}$} & \multicolumn{3}{|c|}{ Placebo } & \multicolumn{4}{|c|}{ Olanzapine } \\
\hline & Baseline & Day 2-4 & Day 28-31 & Baseline & Day 2-4 & Day 28-31 & ANOVA \\
\hline & \multicolumn{3}{|c|}{ Mean \pm SD } & \multicolumn{4}{|c|}{ Mean \pm SD } \\
\hline \multicolumn{8}{|l|}{ SWM } \\
\hline Between errors & $23 \pm 26$ & $22 \pm 23$ & $12 \pm 15$ & $34 \pm 24$ & $31 \pm 19$ & $21 \pm 21$ & $p=.778$ \\
\hline Strategy score & $29 \pm 8.4$ & $29 \pm 7.6$ & $28 \pm 7.8$ & $31 \pm 8.0$ & $30 \pm 7.0$ & $31 \pm 6.9$ & $p=.562$ \\
\hline \multicolumn{8}{|l|}{ RTI } \\
\hline Reaction time & $410 \pm 80.6$ & $395 \pm 43.3$ & $405 \pm 52$ & $410 \pm 110$ & $380 \pm 92.8$ & $388 \pm 111$ & $p=.333$ \\
\hline Movement time & $579 \pm 110$ & $567 \pm 104$ & $539 \pm 61$ & $542 \pm 132$ & $563 \pm 136$ & $530 \pm 107$ & $p=.395$ \\
\hline SSP & $5.8 \pm 1.4$ & $5.7 \pm 1.6$ & $5.6 \pm 1.6$ & $5.4 \pm 1.4$ & $5.4 \pm 1.2$ & $5.7 \pm 1.4$ & $p=.296$ \\
\hline
\end{tabular}

The mean \pm standard deviation (SD) of cognitive measures for both olanzapine and placebo treated group.SWM = Spatial Working Memory task, RTI = Reaction Time task, SSP = Spatial Span task. P value reported for the interaction (time by treatment) of a two-way repeated measures ANOVA.

treatment resistant MDD. They showed significant improvements in sleep efficiency, total sleep time, percentage of time awake, total $\mathrm{N}$ percentage, subjective sleep quality, a decrease in $\mathrm{R}$ percent, and an increased $\mathrm{R}$ latency after a single dose of olanzapine. Our study revealed similar improvements only for sleep continuity measures and illness severity. We observed similar improvements in HDRS scores. The study by Sharpley et al. [13] included only patients with treatment resistant (to SSRI's) MDD, and olanzapine was administered open-label with no control or comparison group. In comparison, in our randomized, double-blind and placebo controlled study, both patients with MDD and BD were included, with most patients that were recommended to receive augmentation to their antidepressant treatment being treatment-resistant to SSRI's. Thus, conflicting findings may be due to the differences in the populations studied and methodology.

Antidepressants rarely improve sleep continuity; most improvements are seen with tricyclic antidepressants. Increased total sleep time and sleep efficiency, as well as decreased sleep latency and total wake time were observed here, similar to that seen with other augmentation agents such as risperidone [64], but different than quetiapine, which does not alter sleep continuity in depression [65]. The beneficial effects of olanzapine treatment on sleep continuity may be due to its diverse pharmacological profile. Primarily, olanzapine is an antagonist at 5-HT2A/C, 5-HT3 and 5-HT6 receptors [33]. A reduction in serotonin firing rates allows for the first sleep cycle to occur and serotonin suppresses $R$ sleep by inhibition of R-promoting cholinergic neurons [6]. Olanzapine may also promote normalization of sleep through antagonism of overactive cholinergic neurons, as olanzapine has affinity for muscarinic M1-M5 receptors. Cholinergic neurons are responsible for the initial activation and ongoing generation of $R$ sleep [6]. Olanzapine also has moderate affinity for the $\alpha$-adrenergic 1 receptor. The adrenergic system plays a role in the suppression of $\mathrm{R}$ and the promotion of sleep cycles [6]. Finally, olanzapine also has affinity for the histamine1 receptor. Histamine is thought to promote wakefulness and reductions in histamine allow for sleep to occur [29]. Therefore, olanzapine's effects on neurotransmitter systems important for normal sleep continuity likely caused improvement in reported sleep continuity.

Few medications have been reliably shown to improve SWS in the treatment of either unipolar or bipolar depression. Only one randomized controlled trial (RCT) examining the effects of trazodone on sleep using polysomnography was identified. A study by Saletu-Zyhlarz et al. [66] examined insomnia patients with dysthymia and reported that trazodone administration significantly increased latency to stage 3 , time and percentage in stage 3, sleep efficiency, and number of awakenings. A significant increase in the amount and percentage of $R$ sleep was also seen. Bemmel et al. [67] conducted a single blind study of trazodone treatment on sleep architecture in patients with MDD. They reported no improvements to SWS and significant $\mathrm{R}$ suppression. In contrast, Mouret et al. [68] examined the effects of open-label trazodone on sleep in patients with MDD and reported significant improvements in SWS and no effect on R sleep. A study by Kluge et al. [69] examined the effects of duloxetine openlabel on sleep in MDD, and reported a significant improvement in latency to SWS and time in SWS. Significant R suppression was reported. No RCTs on the effects of duloxetine were identified. Antidepressants reliably suppress $\mathrm{R}$ sleep; however, their effects on SWS are inconsistent in the treatment of major depressive episodes.

Almost all antidepressants have been shown to suppress $\mathrm{R}$ sleep by increasing the latency to $\mathrm{R}$ and decreasing the total duration of $\mathrm{R}$ sleep. $\mathrm{R}$ suppression was not seen in this study. Although expected, the lack of $\mathrm{R}$ suppression may be due to the concomitant medications of the participants. Only three participants were not taking any other medications, $80 \%$ of participants were taking at least one antidepressant, one participant was taking 
a benzodiazepine only, and one participant was taking lithium only. Over $50 \%$ of participants were taking more than one medication at enrolment into the study. The antidepressant medications and lithium may have suppressed R sleep and thus further suppression of $\mathrm{R}$ sleep with the addition of olanzapine might have been too minimal to detect. Although previous studies of augmentation to SSRIs have shown $\mathrm{R}$ suppression, the degree of polypharmacy was less in these studies, as each participant was only taking one antidepressant [70]. This may reflect the changes often seen with psychotropic treatment to sleep architecture [65,71]. Few medications have been reliably shown to suppress $\mathrm{R}$ sleep and also increase sleep continuity and SWS. Many medications, especially antidepressants, suppress $\mathrm{R}$ sleep and have either no effect or detrimental effects on sleep continuity or SWS. Also, several augmentation strategies, including olanzapine, have been shown to improve sleep continuity and SWS and have no effect on R sleep. Therefore, it is plausible that $\mathrm{R}$ suppression and other changes in sleep architecture are due to separate underlying mechanisms and may need to be treated separately.

The improvement of depressive symptoms observed in this study is representative of the effects of other atypical antipsychotic agents in the treatment of depression, as the olanzapine group had significantly greater improvement than placebo in the MADRS score. The power of the study, however, was not such as to determine efficacy. Clinical response was seen in $46 \%$ of olanzapine-treated patients while only $20 \%$ of placebo-treated patients did so. Twenty-six percent of olanzapine-treated participants reached remission and less than half of that $(10 \%)$ in the placebo group did so. Previously reported response rates in a randomized double-blind study of olanzapine/ fluoxetine combination for the treatment of depression are similar to what is observed here [36]. Significant improvement in depressive symptoms with olanzapine/ fluoxetine combination versus placebo in the treatment of bipolar disorder has been reported as well [72].

The literature on cognitive function in depression is quite diverse and conflicting, as there seems to be no clear pattern of dysfunction in MDD [14,73,74]. The lack of changes seen here in working memory or psychomotor function was not unexpected. Impairments seen in neurocognitive tests in depression may be due to a lack of motivation to complete the task accurately and thus psychotropic treatment or sleep normalization would not have an effect on this beyond improvement of depressive symptoms. As only $26 \%$ of our participants reached remission, many participants had residual depressive symptoms and may not have had an improvement in motivation. The lack of a normal control group precludes us from reporting if the level of baseline functioning is within the normal range or is impaired and did not improve with treatment.
There are a number of limitations in this study. First, participants were maintained on a large variety of concomitant medications. This likely contributed to the highly variable effect of olanzapine on SWS. Secondly, there were a large number of secondary outcome measures and a relatively small sample size. This is most relevant to the lack of change seen in SWS, R sleep and on the neurocognitive measures. A larger sample size may also lend itself to a more precise differentiation between placebo- and olanzapine-treated participants on improvements in subjective sleep quality. Furthermore, the duration of this study only permitted evaluation of the short-term treatment of depression; it is well understood that to observe full improvement with many different psychotropic medications 6-8 weeks or even longer is needed. Finally, the results of this study are generalizable to a select number of patient populations, as there were some comorbid exclusions and a variety of concomitant medications. For instance, the results of this study do not allow us to understand what effect olanzapine may have on sleep architecture in patients with comorbid substance abuse. Further studies should evaluate the influence of different dosages and long-term studies are needed to see if improvements continue into treatment maintenance.

\section{Conclusions}

Olanzapine augmentation in the treatment of both unipolar and bipolar depression resulted in significant improvements in sleep continuity, total sleep time and latency to sleep onset as well as significantly improved depressed mood. However, significant changes were only moderately seen in SWS and not at all in R sleep. Olanzapine has been shown to increase SWS in other psychiatric disorders, e.g., schizophrenia [75]. Factors as simple as dosage of olanzapine may moderate affects on SWS across patient populations. Furthermore, even within patient populations much of the literature shows conflicting results [66-71] of olanzapine on sleep architecture. Changes in cognition, both working memory and psychomotor function, were not observed. However, olanzapine may be one of few medications that improve sleep continuity, thus directly targeting symptoms of depression. Olanzapine may produce this effect through its high affinity for not only serotonin receptors but also muscarinic, adrenergic and histaminergic receptors. The heterogeneity of this study population allows for generalizability to clinical populations, and as such olanzapine may be an effective tool in the treatment of sleep dysfunction in depression.

\section{Abbreviations}

MADRS: Montgomery-asberg depression rating scale; CANTAB: Cambridge neuropsychological test automated battery; SWM: Spatial working memory; SSP: Spatial span; RTI: Reaction time; MDD: Major depressive disorder; BD: Bipolar disorder; R: Rapid eye movement; N: Non-REM; SWS: Slow wave sleep; TST: Total sleep time; EEG: Electroencephalogram; NA: Norepinephrine; 5-HT: Serotonin; MINI: Mini International neuropsychiatric inventory; 
MDE: Major depressive episode; HDRS-17: Hamilton depression rating scale-17; YMRS: Young mania rating scale; HARS: Hamilton anxiety rating scale; PSQI: Pittsburgh sleep quality index; VAS: Visual analogue scale; ESS: Epworth sleepiness scale; CGI-S: Clinical global impression-severity; CGI-I: Clinical global impression-improvement; PSG: Polysomnograph; EMG: Electromyogram; RDI: Respiratory disturbance index; SD: Standard deviation; SSRI: Selective serotonin reuptake inhibitor; RCT: Randomized controlled trial.

\section{Competing interests}

The study was funded by a research fellowship from Eli Lilly Canada as awarded to L. Lazowski under the supervision of Dr. R. Milev. BT, ERH, RJ RT declare that they have no competing interests.

\section{Authors' contributions}

LL contributed substantially to the conception and design, acquisition of data, drafting the article and gave final approval of the version to be published. BT contributed substantially to the analysis and interpretation of the data, drafting the article and gave final approval of the version to be published. ERH contributed substantially to the analysis and interpretation of data, revised it critically for important intellectual content and gave final approval of the version to be published. RJ contributed substantially to the conception and design, acquisition of data, drafting the article and gave final approval of the version to be published. RT contributed substantially to the conception and design, acquisition of data, drafting the article and gave final approval of the version to be published. RM contributed substantially to the conception and design, revised it critically for important intellectual content and gave final approval of the version to be published.

\section{Acknowledgements}

Special thanks to Dr. Lawson for his invaluable statistical advice and support. Thank you to Alan Lowe for project assistance. Thank you to all of the MDRTS staff who made this project possible, especially Teresa Garrah, Judy Joannette, Liane Tackaberry, Ann Shea, Dr. Aldaoud and Dr. DeAngelis, and all of the participants.

\section{Author details}

${ }^{1}$ Centre for Neuroscience Studies, Queen's University, Kingston, Canada.

${ }^{2}$ Department of Psychology, Carleton University, Ottawa, Canada. ${ }^{3}$ Department of Psychiatry, Queen's University, 752 King Street West, Kingston, ON K7L 4X3, Canada.

Received: 17 July 2013 Accepted: 10 July 2014

Published: 17 July 2014

\section{References}

1. Reite M, Ruddy J, Nagel K: Evaluation and Management of Sleep Disorders. 3rd edition. Arlington, VA: American Psychiatric Publishing; 2002

2. Ford DE, Kamerow DB: Epidemiologic study of sleep disturbances and psychiatric disorders: An opportunity for prevention? JAMA 1989, 262:1479-1484.

3. Chang PP, Ford DE, Mead LA, Cooper-Patrick L, Klag MJ: Insomnia in young men and subsequent depression: The Johns Hopkins Precursors Study. Am J Epidemiol 1997, 146:105-114.

4. Dew MA, Reynolds CF III, Houck PR, Hall M, Buysse DJ, Frank E, Kupfer D: Temporal profiles of the course of depression during treatment: Predictors of pathways toward recovery in the elderly. Arch Gen Psychiatry 1997, 54:1016-1024.

5. Reynolds CF III, Frank E, Houck PR, Mazumdar S, Dew MA, Cornes C, Buysse D, Begley A, Kupfer D: Which elderly patients with remitted depression remain well with continued interpersonal psychotherapy after discontinuation of antidepressant medication? Am J Psychiatry 1997, 154:958-962.

6. Markov D, Goldman M: Normal sleep and circadian rhythms: neurobiologic mechanisms underlying sleep and wakefulness. Psychiatr Clin North Am 2006, 29:841-853.

7. Hirshkowitz M: Normal human sleep: An overview. Med Clin North Am 2004, 88:551-565. vii.

8. Riemann D, Berger M, Voderholzer U: Sleep and depression-results from psychobiological studies: an overview. Biol Psychol 2001, 57:67-103.
9. Kupfer DJ, Harrow M, Detre T: Sleep patterns and psychopathology. Acta Psychiatr Scand 1969, 45:75-89.

10. Kupfer DJ, Foster FG: Interval between onset of sleep and rapid-eye-movement sleep as an indicator of depression. Lancet 1972, 300:684-686.

11. Kupfer DJ, Foster FG, Reich L, Thompson SK, Weiss B: EEG sleep changes as predictors in depression. Am J Psychiatry 1976, 133:622-626.

12. Benca RM, Obermeyer WH, Thisted RA, Gillin JC: Sleep and psychiatric disorders: A meta-analysis. Arch Gen Psychiatry 1992, 49:651-668.

13. Sharpley AL, Attenburrow ME, Hafizi S, Cowen PJ: Olanzapine increases slow wave sleep and sleep continuity in SSRI-resistant depressed patients. J Clin Psychiatry 2005, 66:450-454.

14. Sweeney JA, Kmiec JA, Kupfer DJ: Neuropsychologic impairments in bipolar and unipolar mood disorders on the CANTAB neurocognitive battery. Biol Psychiatry 2000, 48:674-684.

15. Gruber S, Rathgeber K, Braunig P, Gauggel S: Stability and course of neuropsychological deficits in manic and depressed bipolar patients compared to patients with Major Depression. J Affect Disord 2007, 104:61-71.

16. Elliott R, Sahakian BJ, McKay AP, Herrod JJ, Robbins TW, Paykel ES: Neuropsychological impairments in unipolar depression: the influence of perceived failure on subsequent performance. Psychol Med 1996, 26:975-989.

17. Grant MM, Thase ME, Sweeney JA: Cognitive disturbance in outpatient depressed younger adults: evidence of modest impairment. Biol Psychiatry 2001, 50:35-43.

18. Purcell $R$, Maruff $P$, Kyrios $M$, Pantelis $C$ : Neuropsychological function in young patients with unipolar major depression. Psychol Med 1997, 27:1277-1285.

19. Nilsson JP, Soderstrom M, Karlsson AU, Lekander M, Akerstedt T, Lindroth $\mathrm{NE}$, Axelsson J: Less effective executive functioning after one night's sleep deprivation. J Sleep Res 2005, 14:1-6.

20. Chee MW, Chuah LY: Functional neuroimaging insights into how sleep and sleep deprivation affect memory and cognition. Curr Opin Neurol 2008, 21:417-423.

21. Jones K, Harrison Y: Frontal lobe function, sleep loss and fragmented sleep. Sleep Med Rev 2001, 5:463-475.

22. Banks S, Dinges DF: Behavioral and physiological consequences of sleep restriction. J Clin Sleep Med 2007, 3:519-528.

23. Ferrara M, De GL, Casagrande M, Bertini M: Selective slow-wave sleep deprivation and time-of-night effects on cognitive performance upon awakening. Psychophysiology 2000, 37:440-446.

24. Ferrara M, De GL, Bertini M: The effects of slow-wave sleep (SWS) deprivation and time of night on behavioral performance upon awakening. Physiol Behav 1999, 68:55-61.

25. Naylor E, Penev PD, Orbeta L, Janssen I, Ortiz R, Colecchia EF, Keng M, Finkel S, Zee PC: Daily social and physical activity increases slow-wave sleep and daytime neuropsychological performance in the elderly. Sleep 2000, 23:87-95.

26. Göder R, Boigs M, Braun S, Friege L, Fritzer G, Aldenhoff JB, Hinze-Selch D: Impairment of visuospatial memory is associated with decreased slow wave sleep in schizophrenia. J Psychiat Res 2004, 38:591-599.

27. John J, Wu MF, Boehmer LN, Siegel JM: Cataplexy-active neurons in the hypothalamus: implications for the role of histamine in sleep and waking behavior. Neuron 2004, 42:619-634.

28. Wu MF, Gulyani SA, Yau E, Mignot E, Phan B, Siegel JM: Locus coeruleus neurons: cessation of activity during cataplexy. Neuroscience 1999, 91:1389-1399.

29. Saper CB, Chou TC, Scammell TE: The sleep switch: hypothalamic control of sleep and wakefulness. Trends Neurosci 2001, 24:726-731.

30. Szymusiak R: Magnocellular nuclei of the basal forebrain: substrates of sleep and arousal regulation. Sleep 1995, 18:478-500.

31. Sharpley AL, Cowen PJ: Effect of pharmacologic treatments on the sleep of depressed patients. Biol Psychiatry 1995, 37:85-98.

32. Shelton RC, Tollefson GD, Tohen M, Stahl S, Gannon KS, Jacobs TG, Buras WR, Bymaster FP, Zhang W, Spencer KA, Feldman PD, Meltzer HY: A novel augmentation strategy for treating resistant major depression. Am Psychiatry 2001, 158:131-134.

33. Bymaster FP, Rasmussen K, Calligaro DO, Nelson DL, DeLapp NW, Wong DT, Moore NA: In vitro and in vivo biochemistry of olanzapine: a novel, atypical antipsychotic drug. J Clin Psychiatry 1997, 58:28-36.

34. Landsness EC, Goldstein MR, Peterson MJ, Tononi G, Benca RM: Antidepressant effects of selective slow wave sleep deprivation in major 
depression: A high-density EEG investigation. J Psychiatr Res 2011, 45:1019-1026.

35. Manber R, Edinger JD, Gress JL, San Pedro-Salcedo MG, Kuo TF, Kalista T: Cognitive behavioural therapy for insomnia enhances depression outcome in patients with comorbid major depressive disorder and insomnia. Sleep 2008, 31:489-495.

36. Thase ME, Corya SA, Osuntokun O, Case M, Henley DB, Sanger TM, Watson SB, Dube S: A randomized, double-blind comparison of olanzapine/fluoxetine combination, olanzapine, and fluoxetine in treatment-resistant major depressive disorder. J Clin Psychiatry 2007, 68:224-236.

37. Buysse DJ, Kupfer DJ, Frank E, Monk TH, Ritenour A, Ehlers C: Electroencephalographic sleep studies in depressed outpatients treated with interpersonal psychotherapy. I. Baseline studies in responders and non-responders. Psychiatry Res 1992, 40:13-26.

38. Salin-Pascual RJ, Galicia-Polo L, Drucker-Colin R: Sleep changes after 4 consecutive days of venlafaxine administration in normal volunteers. J Clin Psychiatry 1997, 58:348-350.

39. Wiegand M, Riemann D, Schreiber W, Lauer CJ, Berger M: Morning and afternoon naps in depressed patients after total sleep deprivation: sleep structure and impact on mood. Biol Psychiatry 1993, 33:467-476.

40. Sharpley AL, Vassallo CM, Cowen PJ: Olanzapine increases slow-wave sleep: evidence for blockade of central $5-\mathrm{HT}(2 \mathrm{C})$ receptors in vivo. Biol Psychiatry 2000, 47:468-470.

41. Lindberg N, Virkkunen M, Tani P, Appelberg B, Virkkala J, Rimon R, Porkka-Heiskanen T: Effect of a single-dose of olanzapine on sleep in healthy females and males. Int Clin Psychopharmacol 2002, 17:177-184.

42. Sharpley AL, Vassallo CM, Pooley EC, Harrison PJ, Cowen PJ: Allelic variation in the $5-\mathrm{HT} 2 \mathrm{C}$ receptor (HT2RC) and the increase in slow wave sleep produced by olanzapine. Psychopharmacology 2001, 153:271-272.

43. Meltzer HY, McGurk SR: The effects of clozapine, risperidone, and olanzapine on cognitive function in schizophrenia. Schizophr Bull 1999 25:233-255.

44. American Psychiatric Association: Diagnostic and Statistical Manual of Mental Disorders. 4th edition. Washington, DC: American Psychiatric Association; 1994.

45. Sheehan DV, Lecrubier $Y$, Sheehan KH, Amorim P, Janavs J, Weiller $E_{r}$ Hergueta T, Baker R, Dunbar G: The Mini-International Neuropsychiatric Interview (M.I.N.I.): the development and validation of a structured diagnostic psychiatric interview for DSM-IV and ICD-10. I Clin Psychiatry 1998, 59:22-33.

46. Young RC, Biggs JT, Ziegler VE, Meyer DA: A rating scale for mania: reliability, validity and sensitivity. Br J Psychiatry 1978, 133:429-435

47. Hamilton M: A rating scale for depression. I Neurol Neurosurg Psychiatry 1960, 23:56-62.

48. Montgomery SA, Asberg M: A new depression scale designed to be sensitive to change. Br J Psychiatry 1979, 134:382-389.

49. Hamilton M: The assessment of anxiety states by rating. $\mathrm{Br} J \mathrm{Med}$ Psychol 1959, 32:50-55.

50. Hamilton M: Diagnosis and rating of anxiety. Br J Psychiatry 1969, Special Pub 3:76-79.

51. Buysse DJ, Reynolds CF III, Monk TH, Berman SR, Kupfer DJ: The Pittsburgh Sleep Quality Index: a new instrument for psychiatric practice and research. Psychiatry Res 1989, 28:193-213.

52. Dixon JS, Bird HA: Reproducibility along a $10 \mathrm{~cm}$ vertical visual analogue scale. Ann Rheum Dis 1981, 40:87-89.

53. Johns MW: A new method for measuring daytime sleepiness: the Epworth sleepiness scale. Sleep 1991, 14:540-545.

54. Guy W: Clinical Global Impressions. In ECDEU Assessment Manual for Psychopharmacology, revised. Edited by Guy W. Rockville, MD: National Institute of Mental Health; 1976.

55. Lawrence AD, Sahakian BJ: The Neuropsychology of Frontostriatal Dementias. In Handbook of the Clinical Psychology of Aging. Edited by Woods RT. New York: Wiley; 1996:243-265.

56. Robbins TW, James M, Owen AM, Sahakian BJ, McInnes L, Rabbitt P: Cambridge Neuropsychological Test Automated Battery (CANTAB): A factor analytic study of a large sample of normal elderly volunteers. Dementia 1994, 5:266-281.

57. Bonnet A, Carley D, Carskadon M, Easton P, Guilleminault C, Harper R, Hayes B, Hirshkowitz M, Ktonas P, Keenan S, Pressman M, Roehers T, Smith J, Walsh J, Weber S, Westbrook P: EEG arousals: scoring rules and examples:
A preliminary report from the Sleep Disorders Atlas Task Force of the American Sleep Disorders Association. Sleep 1992, 15:173-184.

58. Sharpley AL, Williamson DJ, Attenburrow ME, Pearson G, Sargent P, Cowen PJ: The effects of paroxetine and nefazodone on sleep: a placebo controlled trial. Psychopharmacology (Berl) 1996, 126:50-54

59. Sharpley AL, McGavin CL, Whale R, Cowen PJ: Antidepressant-like effect of Hypericum perforatum (St John's wort) on the sleep polysomnogram. Psychopharmacology (Berl) 1998, 139:286-287.

60. Iber C, Ancoli-Israel S, Chesson A, Quan SF: The AASM Manual for the Scoring of Sleep and Associated Events: Rules, Terminology and Technical Specifications. 1st edition. Weschester: American Academy of Sleep Medicine; 2007.

61. Ruehland WR, O'Donoghue FJ, Pierce RJ, Thornton AT, Singh P, Copland JM, Stevens B, Rochford PD: The 2007 AASM recommendations for EEG electrode placement in polysomnography: Impact on sleep and cortical arousal scoring. Sleep 2011, 34:73-81.

62. Gould GA, Whyte KF, Rhind GB, Airlie MAA, Catterall JR, Shapiro CM, Douglas NJ: The sleep hypopnea syndrome. Am J Respir Crit Care Med 1988, 137:895-898.

63. American Academy of Sleep Medicine Task Force: Sleep-related breathing disorders in adults: recommendations for syndrome definition and measurement techniques in clinical research. Sleep 1999, 22:667-689.

64. Cohrs S, Meier A, Neumann AC, Jordan W, Ruther E, Rodenbeck A: Improved sleep continuity and increased slow wave sleep and REM latency during ziprasidone treatment: a randomized, controlled, crossover trial of 12 healthy male subjects. J Clin Psychiatry 2005, 66:989-996.

65. Gedge L, Lazowski L, Murray D, Jokic R, Milev R: Effects of quetiapine on sleep architecture in patients with unipolar or bipolar depression. Neuropsychiatr Dis Treat 2010, 6:501-508

66. Saletu-Zyhlarz GM, Abu-Bakr MH, Anderer P, Semler B, Decker K, Parapatics S, Tschida U, Winkler A, Saletu B: Insomnia related to dysthymia: polysomnographic and psychometric comparison with normal controls and acute therapeutic trials with trazodone. Neuropsychobiology 2001, 44:139-149.

67. Bemmel AL, Havermans RG, Diest R: Effects of trazodone on EEG sleep and clinical state in major depression. Psychopharmacology 1992, 107:569-574.

68. Mouret J, Lemoine P, Minuit MP, Benkelfat C, Renardet M: Effects of trazodone on the sleep of depressed subjects-a polygraphic study. Psychopharmacology (Berl) 1988, 95(Suppl):S37-S43.

69. Kluge M, Schussler P, Steiger A: Duloxetine increases stage 3 sleep and suppresses rapid eye movement (REM) sleep in patients with major depression. Eur Neuropsychopharmacol 2007, 17:527-531.

70. Sharpley AL, Bhagwagar Z, Hafizi S, Whale WR, Gijsman HJ, Cowen PJ: Risperidone augmentation decreases rapid eye movement sleep and decreases wake in treatment-resistant depressed patients. J Clin Psychiatry 2003, 64:192-196.

71. Kupfer DJ, Reynolds CF III, Weiss BL, Foster FG: Lithium carbonate and sleep in affective disorders: further considerations. Arch Gen Psychiatry 1974, 30:79-84.

72. Dube S, Tollefson GD, Thase ME, Briggs SD, Van Campen LE, Case M, Tohen M: Onset of antidepressant effect of olanzapine and olanzapine/fluoxetine combination in bipolar depression. Bipolar Disord 2007, 9:618-627.

73. Taylor Tavares JV, Clark L, Cannon DM, Erickson K, Drevets WC, Sahakian BJ Distinct profiles of neurocognitive function in unmedicated unipolar depression and bipolar II depression. Biol Psychiatry 2007, 62:917-924.

74. Weiland-Fiedler P, Erickson K, Waldeck T, Luckenbaugh DA, Pike D, Bonne O, Charney DS, Neumeister A: Evidence for continuing neuropsychological impairments in depression. J Affect Disord 2004, 82:253-258.

75. Kluge M, Schacht A, Himmerrich H, Rummel-Kluge C, Wehmeier PM, Dalah M, Hinze-Selch D, Kraus T, Dittmann RW, Pollmacher T, Schuld A: Olanazpine and clozapine differently affect sleep in patients with schizoprhrenia: Results from a double-blind, polysomnographic study and review of the literature. Schizophr Res 2014, 152:255-260.

doi:10.1186/1471-244X-14-202

Cite this article as: Lazowski et al:: Sleep architecture and cognitive changes in olanzapine-treated patients with depression: A double blind randomized placebo controlled trial. BMC Psychiatry 2014 14:202. 